\title{
DECENTRALIZED “GOOD NEIGHBOR” DSA BASED ON ADAPTIVE ANTENNA ARRAY INTERFERENCE MITIGATION DIVERSITY: FINITE AMOUNT OF DATA EFFECTS
}

\author{
Alexandr M. Kuzminskiy*
}

Alcatel-Lucent

The Quadrant, Swindon SN5 7DJ, UK

Alexandr.Kuzminskiy@alcatel-lucent.com

\author{
Yuri I. Abramovich
}

\author{
Defence Science and Technology Organization \\ PO Box 1500, Edinburgh SA 5111, Australia \\ Yuri.Abramovich@dsto.defence.gov.au
}

\begin{abstract}
Finite amount of data spectrum sensing effects are addressed in decentralized dynamic spectrum allocation (DSA) that exploits adaptive antenna array interference mitigation (IM) diversity at the receiver. The system is based on spectrally efficient filter bank multicarrier (FBMC) PHY and consists of base stations (BSs) that use a "good neighbor" rule-regulated strategy to optimize uplink frequency allocation to their subscriber stations (SSs) to achieve the least impact of IM on the useful signal. A spectrum sensing protocol is proposed and DSA efficiency is studied by means of semianalytical Markov chain approach for low-dimension networks and statistical simulations for higher-dimension spectrum sharing systems.
\end{abstract}

Index Terms - Decentralized dynamic spectrum allocation, interference mitigation, "good neighbor" strategy, Markov chain.

\section{INTRODUCTION}

Dynamic spectrum allocation (DSA) is an effective way to increase the spectral efficiency of wireless communications systems, e.g., [1], and others. DSA can be implemented using explicit coordination between access nodes, which is mostly suitable for cellular systems in a licensed spectrum. However, in an unlicensed spectrum, frequency band allocation has to be performed by each provider in a decentralized autonomous way, e.g., as in [2]. In the most challenging interference-limited scenario, where the total number of SSs that belong to different closely located subsystems exceeds the number of available bands, joint DSA and multiple-antenna interference suppression is required, e.g., [3], and others.

One example of decentralized unsynchronized scenario based on OFDM physical layer could be a number of fixed WIMAX systems sharing a license exempt spectrum [2]. The main feature of the OFDM physical layer in this case is that guard bands are required between frequency bands allocated to users from different unsynchronized subsystems to prevent energy leakage between them as illustrated in Fig. 1 in the 3-band case. Particularly, the fixed WIMAX standard defines the OFDM physical layer with totally $F_{\text {total }}=256$ sub-carriers, $F_{\text {guard }}=27$ sub-carriers guard sub-carriers and minimum 10 sub-carriers in a frequency band. This means that maximum $F=6$ bands for unsynchronized users can be formed [2] and their

\footnotetext{
*Part of this work has been done in the context of the IST FP7 PHYDYAS project.
}

spectrum efficiency in terms of spectrum utilization defined as

$$
\rho(F)=1-\frac{(1+F) F_{\text {guard }}}{F_{\text {total }}},
$$

is shown in Fig. 2.

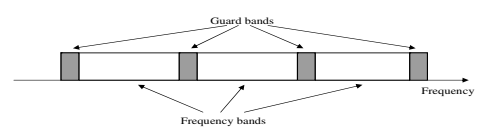

Fig. 1. Frequency bands for unsynchronized users

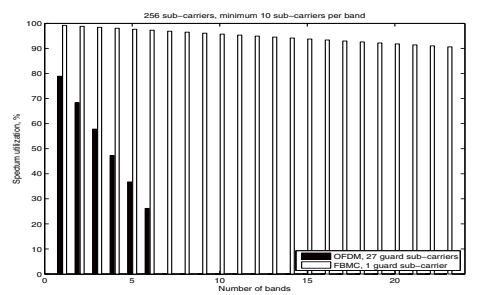

Fig. 2. Spectrum utilization for unsynchronized systems sharing license exempt spectrum

One can see in Fig. 2 that only about $26 \%$ of spectrum can be used for data transmission in the 6-band case. The rest of the spectrum is wasted for guard bands in this system. Obviously, OFDM physical layer is not a spectrally efficient solution in this situation. Another physical layer with reduced $F_{\text {guard }}$ could be more efficient in the asynchronous cognitive radio (CR) scenario. FBMC PHY can be considered as such a candidate [4]. Particularly, if FBMC could allow $F_{\text {guard }}=1$, then up to 23 bands with spectrum efficiency above $90 \%$ could be formed in the same scenario as also illustrated in Fig. 2. Obviously, this is just an illustration example to emphasize the physical layer importance in the CR scenario. In practice, guard bands for both OFDM and FBMC depend on particular power density spectrum, RF imperfections, system parameters, etc., [4]. Furthermore, physical layer features affect spectrum sensing and other basic CR operations as also studied in [4]

Spectrum sharing "good neighbor" (GN) approach for FBMC based networks is introduced in [4], [5], which can be considered as rule-regulated cooperation between spectrum sharing subsystems 
without explicit data exchange between them. The antenna array interference mitigation (IM) based DSA algorithms are studied in [4], [5] by means of the theory of absorbing Markov chains and simulations assuming that local second-order statistics is known at each subsystem. In this paper, we introduce spectrum sensing algorithms at each spectrum sharing subsystem to estimate these statistics and study finite amount of data effects in the GN IM-based DSA.

\section{SYSTEM MODEL AND PROBLEM FORMULATION}

The considered system consists of $N$ independent subsystems containing base stations $\mathrm{BS}_{n}, n=1, \ldots, N$ and corresponding users $\mathrm{SS}_{n m}, m=1, \ldots, M$, where $M$ is the number users per BS. A system model is illustrated in Fig. 3 for $N=M=3$. Each user transmits data to its BS using one of the $M \leq F<N M$ available frequency bands. All BSs have full information and control of their own users. In particular, they can estimate propagation channels in all the available bands and assign the individual bands and transmit powers to their own users. Assuming for simplicity narrowband reception, the signal received by an antenna array of $K$ elements for the $n$th subsystem can be expressed as follows:

$$
\mathbf{x}_{n f}(t)=\sum_{m=1}^{M} \delta_{f d_{n m}} q_{n m} \mathbf{h}_{d_{n m} m n n} s_{n m}(t)+\mathbf{z}_{n f}(t),
$$

where $n=1, \ldots, N, f=1, \ldots, F, \mathbf{x}_{n f}(t)$ is the $K \times 1$ vector of the signal received at $\mathrm{BS}_{n}$ in the $f$ th band at the $t$ th time instant, $\mathbf{h}_{f m l n}$ is the $K \times 1$ vector of propagation channel to $\mathrm{BS}_{n}$ in the $f$ th band from the $m$ th user of the $l$ th subsystem, $s_{n m}(t)$ is the $\mathrm{SS}_{n m}$ transmitted signal with $\mathrm{E}\left\{\left|s_{n m}(t)\right|^{2}\right\}=1$ and $q_{n m}^{2}$ is its power with constraint $\sum_{m=1}^{M} q_{n m}^{2}=Q, n=1, \ldots, N, \mathbf{z}_{n f}(t)$ is a $K \times 1$ vector of interference plus AWGN with variance $\sigma^{2}, d_{n m}$ is the $n m$ th element of the $N \times M$ decision matrix $\mathbf{D}$ denoting the frequency band assigned to $\mathrm{SS}_{n m}, \mathrm{E}\{\cdot\}$ is the averaging operator, $(\cdot)^{*}$ is the conjugate transpose operation, $\mathbf{I}_{K}$ is the $K \times K$ unity matrix, and $\delta_{i j}$ is the Kronecker delta function.

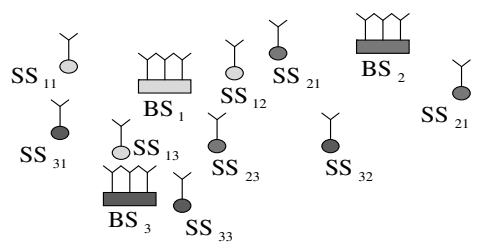

Fig. 3. System model for $N=M=3$

The $K \times K$ interference plus noise covariance matrix at $\mathrm{BS}_{n}$ in the $f$ th band can be expressed as

$$
\mathbf{R}_{n f}=\sum_{l \neq n}^{N} \sum_{m=1}^{M} \delta_{f d_{l m}} q_{l m}^{2} \mathbf{h}_{d_{l m} m l n} \mathbf{h}_{d_{l m} m l n}^{*}+\sigma^{2} \mathbf{I}_{K} .
$$

Note that (3) describes the actual structure of the covariance matrix, which remains unknown at $\mathrm{BS}_{n}$ even if the entire covariance matrix is properly estimated, e.g., from the sensing interval without the desired signal.

A performance metric, which cannot be estimated locally at each $\mathrm{BS}_{n}$, is defined as the data rate for the weakest link in the system

$$
\gamma=\min _{m=1, \ldots, M, n=1, \ldots, N} \log _{2}[1+\operatorname{SINR}(\mathbf{D})],
$$

where

$$
\operatorname{SINR}(\mathbf{D})=\mathbf{h}_{d_{n m} m n n}^{*} \mathbf{R}_{n d_{n m}}^{-1} \mathbf{h}_{d_{n m} m n n}
$$

is the SINR at the output of the optimal spatial filter for the $n m$ th user.

The main system model assumptions are as follows:

- BSs allocate different bands for all users in their subsystems, i.e., all rows in matrix $\mathbf{D}$ contain different elements.

- Rayleigh propagation channels $\mathbf{h}_{f m l n}$ are stationary independent random Gaussian vectors $\mathbf{h} \sim \mathcal{C N}\left(0, \sigma_{h}^{2} \mathbf{I}_{K}\right)$, where $\sigma_{h}^{2}=1$ is assumed to emphasize the interference limited scenario. The impact of random $\sigma_{h}^{2}$ according to the pathloss and shadowing model for the particular system geometries is studied in [5].

- For simplification, the constant power $q_{n m}^{2}=Q / M$ for all users in the system is assumed, i.e., locally selected frequency bands are the only adjustable parameters. A power control in the IM-based DSA networks is addressed in [4], [5].

The GN IM-based DSA algorithm [5] with minimum switch (MinSwitch)search is assumed at $\mathrm{BS}_{n}$ sensing intervals:

$$
\mathbf{d}_{n}=\arg \min _{m, q \in \mathcal{M}, f_{m} \in \mathcal{F}, f_{m} \neq f_{q}, q \neq m} \sum_{m=1}^{M}\left|\operatorname{sign}\left(f_{m}-d_{n m}^{(0)}\right)\right|,
$$

subject to

$$
\log _{2}\left(1+\mathbf{h}_{f_{m} m n n}^{*} \mathbf{R}_{f_{m} n}^{-1} \mathbf{h}_{f_{m} m n n}\right) \geq \gamma_{0}
$$

where $\gamma_{0}$ is the target performance threshold ${ }^{1}, d_{n m}^{(0)}$ is the $m$ th element of the current band allocation vector $\mathbf{d}_{n}^{(0)}$ before the next sensing interval at $\mathrm{BS}_{n}$, and $\operatorname{sign}(a)=\{-1,0,1\}$.

The main assumption in [5] is that all subsystems know their own second order statistics, i.e.,

- $\mathrm{BS}_{n}, n=1, \ldots, N$ know $\mathbf{R}_{n f}$ and $\mathbf{h}_{f m n n}$ for $f=$ $1, \ldots, F, m=1, \ldots, M$, and have no information on $\mathbf{R}_{l f}, \mathbf{h}_{\text {fmnl }}$ for $l \neq n$.

Now, we concentrate on the finite amount of data spectrum sensing effects and assume that

- Space-time spectrum sensing is implemented at each $\mathrm{BS}_{n}$ to estimate the weight vectors and SINR in all the available bands. To do this, we assume that users transmit the pilot symbols during the sensing intervals and data signals during the data intervals. Furthermore, focusing on the cognitive radio effects, we assume that the sensing intervals for different subsystems do not overlap. A low probability of overlapping of the sensing intervals can be achieved, for example, by means of random duration of the data intervals [4].

The following problems need to be addressed:

- modify the basic GN algorithm using estimated second-order statistics,

- design a space-time spectrum sensing protocol that allows such estimation,

- study the IM-based DSA algorithms taking into account the finite amount of data effects,

- propose and investigate modified spectrally efficient IM-based DSA algorithms that allow minimization of the required amount of the training data.

\footnotetext{
${ }^{1}$ The on-line selection of $\gamma_{0}$ in the general case of random $\sigma_{h}^{2}$ for particular system geometries is addressed in [5].
} 


\section{GN IM-BASED DSA WITH ESTIMATED SECOND-ORDER STATISTICS}

The main difference compared with the case of known second-order statistics is that the weight vectors and IM efficiency in terns of SINR or potential data rates have to be estimated over the final number of pilot symbols. Assuming that $T$ pilots are available for each user during a sensing interval for the $n$th subsystem and taking into account that $\sigma_{h}^{2}=1$, the weight vector and SINR can be estimated for $m=1, \ldots, M$ as follows:

$$
\begin{gathered}
\hat{\mathbf{w}}_{n m}=\hat{\mathbf{R}}_{d_{n m} n}^{-1} \hat{\mathbf{g}}_{d_{n m} n}, \\
\hat{\nu}_{n m}=\operatorname{SINR}_{n m}=\left(1-\hat{\mathbf{g}}_{d_{n m} n}^{*} \hat{\mathbf{R}}_{d_{n m} n}^{-1} \hat{\mathbf{g}}_{d_{n m} n}\right)^{-1},
\end{gathered}
$$

where $\hat{\mathbf{R}}_{f n}=T^{-1} \sum_{t=1}^{T} \mathbf{x}_{f n}(t) \mathbf{x}_{f n}^{*}(t), \hat{\mathbf{g}}_{f n}=T^{-1} \sum_{t=1}^{T}$ $\mathbf{x}_{f n}(t) s_{\mathrm{p}}^{*}(t), \hat{\mathbf{g}}_{f n}$ is the estimated propagation channel $\mathbf{h}_{f m n n}$ of the $m$ th user in the $f$ th band, $s \mathrm{p}(t)$ is the pilot symbol, $\mathbf{x}_{f n}(t)$ is the $K \times 1$ vector of the received signal in the $f$ th band.

Then, the GN algorithm with estimated second-order statistics can be expressed as follows:

- Sensing interval

Step 1: For the current band allocation $d_{n m}^{(0)}$ calculate

$$
\hat{\gamma}_{n}=\log _{2}\left(1+\min _{m=1, \ldots, M} \hat{\nu}_{n m}\right)
$$

using (10). If $\hat{\gamma}_{n} \geq \hat{\gamma}_{0}$, where $\hat{\gamma}_{0}$ is the threshold that takes into account the finite amount of data effects (to be defined later), then go to the "Data interval" stage without updating $\mathbf{d}_{n}$, otherwise, go to Step 2;

Step 2: Update the band allocation vector $\mathbf{d}_{n}$ according to (6) subject to $\log _{2}\left(1+\hat{\nu}_{n m}\right) \geq \hat{\gamma}_{0}$.

Step 3: Calculate the optimal weight vector according to (8).

- Data interval

$\mathrm{SS}_{n} m, m=1, \ldots, M$ transmit data in bands assigned in $\mathbf{d}_{n}$;

$\mathrm{BS}_{n}$ receives data with the optimal weight vectors (9).

Now, we need to design a spectrum sensing protocol that allows implementation of the presented GN algorithm. For simplification we assume that users can simultaneously transmit the same pilots in all available bands. Furthermore, we note that transmission of the pilots in the currently occupied bands does not change the interference environment for other subsystems. On the contrary, pilot transmission in all available bands does change the interference environment. This means that pilot transmission in the all the available bands must be avoided in the stationary regime after convergence of the decentralized DSA network.

The proposed spectrum sensing protocol is shown in Fig. 4. At the beginning of the sensing interval all users of the sensing subsystem transmit $T$ pilots in their currently allocated bands without changing the interference scenario for other subsystems. If SINR estimated over the training interval is above the threshold, then all users transmit $T M$ data symbols, again without any changes to the interference environment. If the estimated SINR is below the threshold, then all users sequentially transmit pilots in all available bands to allow estimation of the decision statistics to apply the MinSwitch search in the GN algorithm. In this case, the interference environment is affected, but this is expected anyway because of updates in band allocation in the end of spectrum sensing interval.

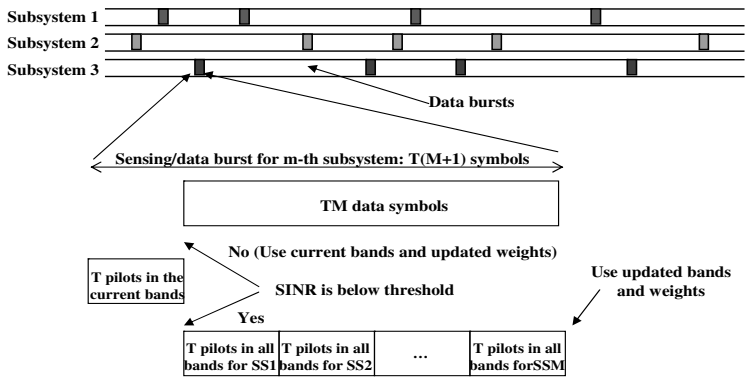

Fig. 4. Space-time spectrum sensing protocol

The total duration of the sensing interval during convergence stage is $T_{\text {tot }}=T(M+1)$ symbols. For high number of users, the required overhead may be quite high. In this situation, development of efficient algorithms that allow reduction of $T$ and correspondingly $T_{\text {tot }}$, becomes critically important. To develop such algorithms, first, we need to study the GN DSA performance depending of the number of pilots $T$.

\section{MARKOV CHAIN ANALYSIS FOR LOW-DIMENSION NETWORKS}

The main difference of the considered finite amount of data case compared to the known second order statistics situation in [5] is that band allocation decision has to be taken based on the SINR (or potential data rate) estimated over $T$ pilot symbols. Clearly, this significantly changes the threshold requirements and Markov chain model developed in [5]. To specify these changes, in [6], we have derived statistical distributions of $\hat{\eta}=\hat{\nu} / \hat{\nu}_{\text {opt }}$, where $\hat{\nu}$ is defined in (9) and $\hat{\nu}_{\text {opt }}$ is the SINR for the actual second-order statistics:

$$
\begin{gathered}
\hat{\eta}=\frac{T}{d_{11}}, w\left(d_{11}\right)=\frac{1}{\Gamma(T-K)} d_{11}^{T-K-1} e^{-d_{11},} \\
\mathrm{E}\left(\hat{\eta}^{l}\right)=\frac{T^{l}(T-K-1-l) !}{(T-K-1) !}, \\
\operatorname{Prob}(\hat{\eta}<\delta)=\beta(\delta, T, K)=e^{-\frac{T}{\delta}} \sum_{t=0}^{T-K-1} \frac{1}{t !}\left(\frac{T}{\delta}\right)^{l},
\end{gathered}
$$

where $\Gamma$ is the gamma function, $w\left(d_{11}\right)$ is p.d.f. of $d_{11}$ and $\beta$ is c.d.f. of $\hat{\eta}$.

Fig. 5 illustrates c.d.f. (13). One can see that the SINR estimated over a short training interval is very likely to be much higher than the actual SINR for the known second order statistics. Now, we can study these effects analytically using the estimated SINR statistics (11)-(13).

In [5], Markov chain analysis of the IM-based DSA with the known local second order statistics is based on the absorbing Markov chain model, which assumes that the absorbing states exist with zero probability to leave them if they are reached from some transient states. It is clear that the considered case of estimated second order statistics significantly changes the underlying Markov chain model.

As in [5], to formulate Markov model we assume that all possible $I=\left(A_{M}^{F}\right)^{N}, A_{M}^{F}=F ! /(F-M)$ ! different allocation matrices $\mathbf{D}_{i}, i=1, \ldots, I$ form states of the Markov chain. At each sensing interval, we assume that one randomly selected subsystem is sensed 
with probability $p_{\text {sens }}=N^{-1}$. Then, the $I \times I$ transition probability matrix $\mathbf{P}=\left\{p_{i j}\right\}$ can be defined as generalization of the similar procedure [5] for the locally known second order statistics:

$$
p_{i j}=p_{0}(i) \delta_{i j}+p_{\text {sens }}\left[1-p_{0}(i)\right] \sum_{n=1}^{N} \sum_{q=1}^{Q} p_{1}(i, n, q) \delta_{j j_{i n q}},
$$

where $Q=A_{M}^{F}$ is the number of all possible band allocations for one subsystem; $p_{0}(i)$ is the probability that $\hat{\gamma}_{n}(i) \geq \hat{\gamma}_{0}$ for all $n=$ $1, \ldots, N$, where $\hat{\gamma}_{n}(i)$ is defined in (10) for the $i$ th state; $p_{1}(i, n, q)$ is the probability to select the $q$ th out of $Q$ possible band allocations as the outcome of sensing at the $n$th subsystem for the $i$ th current state; $j_{i n q}$ is the corresponding state.

Taking into account that if $a_{i} \geq \alpha$ for $i \in \mathcal{I}$ then $\min _{i \in \mathcal{I}} \geq \alpha$, $p_{0}(i)$ can be found using (13):

$$
p_{0}(i)=\prod_{n=1}^{N} \prod_{m=1}^{M}\left[1-\beta\left(\frac{\hat{\nu_{0}}}{\hat{\nu}_{n m}(i)}, T, K\right)\right],
$$

where $\hat{\nu}_{0}=2^{\hat{\gamma}_{0}}-1$ is the SINR threshold.

Unfortunately, it is difficult to get an analytical expression for $p_{1}(i, n, q)$ in (14) because it involves a constrained search according to the GN algorithm. One option could be to use a semi-analytical approach, which is based on the observation that (11) actually is the $\mathcal{X}^{2}$ distribution with $2(T-K)$ degrees of freedom. This means that $d_{11}$ can be simulated as $d_{11}=\sum_{j=1}^{T-K}\left|z_{j}\right|^{2}$, where $z_{j} \sim \mathcal{C N}(0,1)$ are independent random complex Gaussian variables with zero mean and unit power. Then, $p_{1}(i, n, q)$ can be estimated by means of averaging over $J \gg 1$ trials in which the estimated SINR is simulated as $\hat{\nu}=\nu \frac{T}{d_{11}}$, where $\nu$ is the SINR calculated for the actual second order statistics. In each trial, the MinSwitch search of the GN algorithm for the given $\mathbf{D}_{i}$ and the $n$th sensing subsystem can be applied to get the $F \times M$ matrix of estimated SINRs. Then, $p_{1}(i, n, q)$ for the $i_{\text {inq }}$ th state can be calculated by averaging over all $J$ trials.

Now, the transition probability matrix is a sparse $I \times I$ stochastic matrix with maximum $A_{M}^{F} N-N+1$ nonzero elements in a row, such that $\sum_{j=1}^{I} p_{i j}=1$ for $i=1, \ldots, I$, which completely defines Markov model of the considered system.

The main difference compared with the case of the known second-order statistics is that the number of nonzero elements is much higher in the estimated case and the absorbing points formally disappear in the corresponding Markov chain because $\beta(\delta)>0$ for $\delta>0$, although, $\beta(\delta)$ may be very low for $\delta<1$ as can be seen in (13) and Fig. 5.

An illustration of this situation is presented in Fig. 6, which shows c.d.f. of $\alpha=1-p_{0}$, where $p_{0}$ is defined in (15) for the states that are the absorbing ones in the actual transition matrix for $T=12, N=5, M=2, F=3, K=4, \sigma_{s}^{2}=1, \mathrm{SNR}=20 \mathrm{~dB}$, $\gamma_{0}=5 \mathrm{bits} / \mathrm{symbol}$, and $J=1000$ with the following threshold

$$
\hat{\gamma}_{0}=\log _{2}\left[1+\frac{T\left(2^{\gamma_{0}}-1\right)}{T-K-1}\right],
$$

where $T /(T-K-1)$ is the mean value of the relative estimated SINR according to (12).

In fact, Fig. 6 presents c.d.f. of the probability to leave the states corresponding to the absorbing states calculated over 200 hundred independent channel realizations for different numbers of pilot symbols in the spectrum sensing protocol. One can see in Fig. 7 that although probability to leave many of the "actual" absorbing states may be very high, some of them remain very close to absorbing ones. For example, for $T=12$ almost $5 \%$ of the "actual" absorbing states have probability to leave less than $10^{-2}$, and for $T=48$ almost $15 \%$ of these states have less than $10^{-6}$ probability to leave.

The fact that some of the states of the defined Markov chain may be very close to absorbing points in terms of $p_{i i}>1-\alpha, \alpha \ll 1$, but $p_{i i}<1$ for all $i=1, \ldots, I$, means that the theory of absorbing Markov chains [7] is formally not applicable any more. At the same time, the theory of ergodic Markov chains [8] is not practically applicable as well because some elements of the transition probability matrix may be very small that makes practically impossible finding and physical interpretation of the fixed vector as well as other characteristics of the chain in almost singular situations.

Our proposal to overcome this difficulty is to consider a simplified Markov chain modeling, where the states with $p_{i i} \geq 1-\alpha_{0}$ for the given $\alpha_{0} \ll 1$ are replaced with the absorbing states with $p_{i i}=1$. This simplification allows us to apply the theory of absorbing Markov chains [7] to the simplified models similarly to [5], calculate the corresponding fundamental matrices, and eventually find all ergodic subchains (if they exist) and average absorption times for all the initial states. In the considered application, this performance can be interpreted as the average number of sensing intervals before reaching the states with probability to leave them less than $\alpha_{0} \ll 1$.

C.d.f. of the average absorption time for the particular simplified Markov chain for $\alpha_{0}=10^{-3}$ is presented in Fig. 7. One can see that convergence from some initial states still can be very fast, e.g., for $10 \%$ of the initial transient states it is less than 80 iterations on average. Other initial states may experience much slower convergence. Particularly, $20 \%$ of initializations require $10^{4}$ and more iterations to reach states with probability to leave less than $10^{-3}$ in this scenario. A typical example of long-term behavior of the GN algorithm in this scenario is illustrated in Fig. 8 in the same format as in [5]. One can see a very slow convergence to the state with very low probability to leave. This state is marked "absorbing" in Fig. 8, but it is important to emphasize that the system will leave it eventually because of the estimation effects if it is observed sufficiently long time. Although the data rate picture in Fig. 8 (left) looks good because of relatively long stationary intervals, the zoom in Fig. 8 (right) shows that actually, the system demonstrates an undesirable transient long-term behavior before reaching the "absorbing" point leading to some performance degradation. This performance degradation is clearly initiated by the finite amount of data effects in the considered stationary propagation conditions.

Generally, the presented simplified absorbing Markov chain modeling allows a quantitative and qualitative analysis of the final amount of data effects in low-dimensional IM-based DSA networks. Higher-dimension networks can be studied by means of simulations still taking into account the effects and trends established by the proposed analysis.

\section{SIMULATION RESULTS FOR HIGHER-DIMENSION NETWORKS}

The long-term transient behavior because of the estimation effects predicted by the Markov chain analysis in Section 4 and illustrated in Fig. 8 for low dimension networks can be expected for more complicated systems as well. Fig. 9 presents the minimum data rate simulation results over 1000 data intervals in 100 stationary channel re- 
alizations for $N=5, M=5, F=7, K=4, \sigma_{s}^{2}=1$, SNR=20dB, $\gamma_{0}=5 \mathrm{bits} / \mathrm{symbol}$, and variable $T$. One can see that the spectrum sensing effects make the critical impact on the network performance. Particularly, increasing the pilot interval by 2 times from $T=6$ to $T=12$ reduces the number of data intervals with minimum data rate below 4.5 bits/symbol from $90 \%$ to $5 \%$. Obviously, the doubled overhead is the price to be paid for this performance improvement. Further performance improvement requires even more pilot symbols leading to spectrum efficiency degradation. Adaptive averaging over a variable number of sensing intervals proposed in [6] could be a solution to this problem, but this is out of the scope of this paper.

\section{CONCLUSION}

Markov chain analysis and statistical simulations have been applied to investigate of finite amount of data spectrum sensing effects in decentralized "good neighbor" DSA in spectrum sharing wireless networks based on FBMC PHY developed at the PHYDYAS project.

\section{REFERENCES}

[1] D. Gesbert, S. G. Kiani, A. Gjendemsjo, G. E. Oien, "Adaptation, coordination, and distributed resource allocation in interference-limited wireless networks," Proceedings of the IEEE, vol. 95, no. 12, pp. 2393-2409, Dec. 2007.

[2] O. Ashagi, S. Murphy, L. Murphy, "A distributed approach to interference mitigation between OFDM-based 802.16 systems operating in license-exempt spectrum," in Proc. ICC, pp.48554860, June 2007.

[3] G. Scutari, D. Palomar, S. Barbarossa, "Cognitive MIMO radio," IEEE Signal Proc. Magazine, pp. 46-59, Nov. 2008.

[4] "Application of the FBMC physical layer in a cognitive radio scenario," IST FP7 PHYDYAS, INFSO-ICT-211887, D8.1, July 2009, available: http://www.ict-phydyas.org.

[5] A. M. Kuzminskiy, Y. I. Abramovich, "Decentralized dynamic spectrum allocation based on adaptive antenna array interference mitigation diversity," IEEE Trans. Sig. Proc., vol. 58, no. 4, pp. 2246-2260, Apr. 2010.

[6] "Space-time spectrum sensing, algorithms and software description,” IST FP7 PHYDYAS, INFSO-ICT-211887, D8.2, pp. 7698, Feb. 2010, to be available: http://www.ict-phydyas.org.

[7] J. G. Kemeny, G. L. Thompson, Introduction to finite mathematics, Prentice-Hall, N. J., 1966.

[8] W. Feller, "An introduction to probability theory and its applications," John Wiley \& Sons, Inc., 1957.

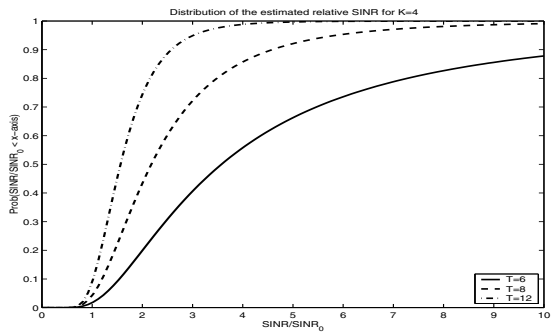

Fig. 5. C.d.f. of $\hat{\eta}$ for $K=4$ and $T=[6,8,12]$

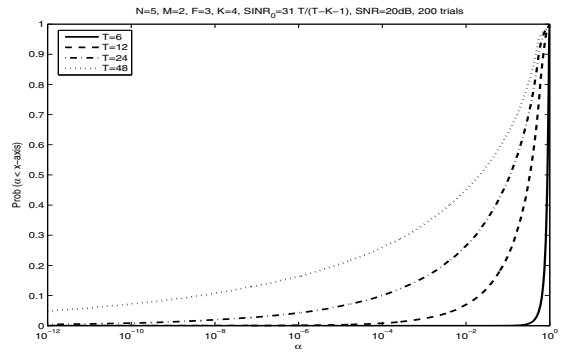

Fig. 6. Probability to leave the states corresponding to the actual absorbing points

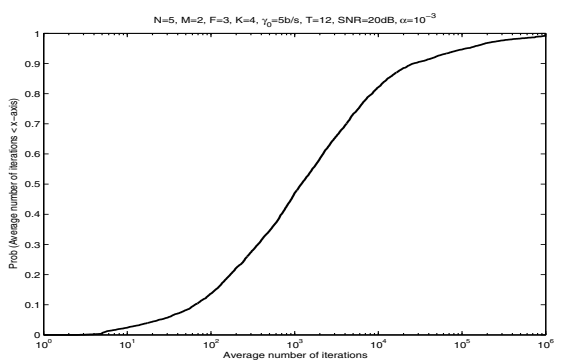

Fig. 7. C.d.f. of the average number of sensing intervals before reaching the states with probability to leave them less than $10^{-3}$
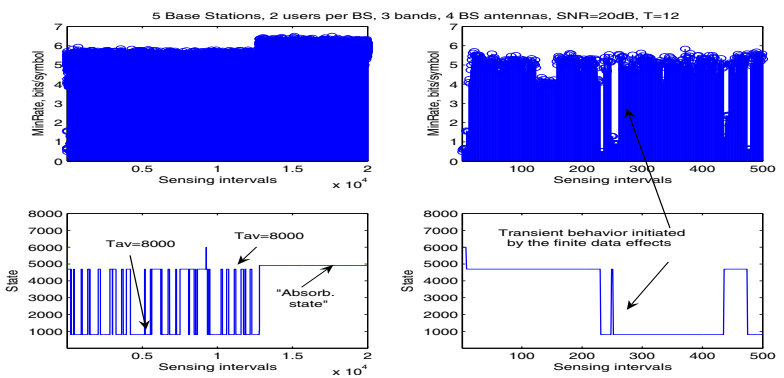

Fig. 8. Typical example of long-term behavior (left) and its initial fragment(zoom)

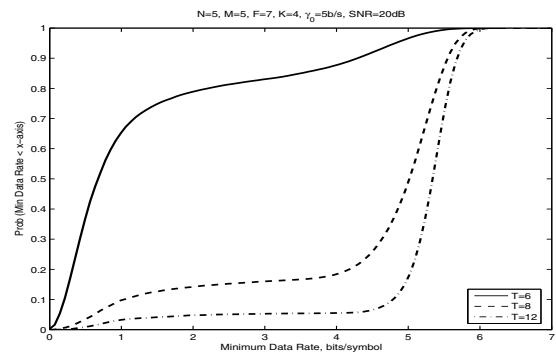

Fig. 9. Simulation results for higher-dimension network 